\title{
Novel Sources of Resistance to Fusarium oxysporum f. sp. lycopersici Race 3 Among Solanum pennellii Accessions
}

\author{
Jian Li, Jessica Chitwood-Brown, and Gurleen Kaur \\ Horticultural Sciences Department, University of Florida, Gainesville, FL 32611
}

\author{
Joanne A. Labate \\ Plant Genetic Resources Unit, U.S. Department of Agriculture, Agricultural Research Service, \\ 630 W. North Street, Geneva, NY 14456
}

G.E. Vallad

Gulf Coast Research and Education Center, University of Florida, Wimauma, FL 33598; and Plant Pathology Department, University of Florida, Gainesville, FL 32611

\author{
Tong Geon Lee \\ Horticultural Sciences Department, University of Florida, Gainesville, FL 32611; Gulf Coast \\ Research and Education Center, University of Florida, Wimauma, FL 33598; and Plant \\ Molecular and Cellular Biology Graduate Program, University of Florida, Gainesville, FL 32611
Samuel F. Hutton Research and Education Center, University of Florida, Wimauma, FL 33598 \\ Horticultural Sciences Department, University of Florida, Gainesville, FL 32611; and Gulf Coast
}

\begin{abstract}
AdDitional INDEX wORDs. fusarium wilt, Solanum lycopersicum, tomato, durable resistance, gene pyramiding
Abstract. Fusarium wilt of tomato (Solanum lycopersicum), caused by fungal pathogen Fusarium oxysporum f. sp. lycopersici $(\mathrm{Fol})$, is one of the most important diseases in tomato production. Three races of the pathogen are described, and race-specific resistance genes have been applied in commercial tomato cultivars for controlling the disease. Race 3 (Fol3) threatens tomato production in many regions around the world, and novel resistance resources could expand the diversity and durability of $\mathrm{Fol}$ resistance. The wild tomato species, Solanum pennellii, is reported to harbor broad resistance to $\mathrm{Fol}$ and was the source of two known Fol3 resistance genes. In this study, we evaluated $42 S$. pennellii accessions for resistance to each fusarium wilt race. $F_{1}$ plants, developed from crossing each accession with the Fol3 susceptible line 'Suncoast', were evaluated for $\mathrm{Fol} 3$ resistance, and $\mathrm{BC}_{1} \mathrm{~F}_{1}$ plants were screened to determine the likelihood that $\mathrm{Fol} 3$ resistance was based on a novel locus (loci). Nearly all accessions showed resistance to Fol3, and many accessions were resistant to all races. Evaluation of $F_{1}$ plants indicated a dominant resistance effect to Fol3 from most accessions. Genetic analysis indicated 24 accessions are expected to contain one or more novel Fol3 resistance loci other than an allele near the $I-3$ locus. To investigate genetic structure of the $S$. pennellii accessions used in this study, we genotyped all 42 accessions using genotyping by sequencing. Approximately $20 \%$ of the single nucleotide polymorphism (SNP) loci were heterozygous across accessions, likely due to the outcrossing nature of the species. Genetic structure analysis at 49,120 unique SNP loci across accessions identified small but obvious genetic differentiations.
\end{abstract}

Fusarium wilt, caused by the fungal pathogen Fusarium oxysporum f. sp. lycopersici $(\mathrm{Fol})$, is a serious disease of tomato (Solanum lycopersicum) grown in warm production regions. The pathogen penetrates the root and infects the vascular system, blocking the supply of water and nutrition, and results in wilting, stunting, and, ultimately, plant death. The disease can cause devastating crop losses, and heavily infested fields were often abandoned before the availability of resistant cultivars (McGrath et al., 1987; Scott and Jones, 1989). Cultural practices, such as raising the soil $\mathrm{pH}$ level, using nitrate-nitrogen, and exercising sanitary

Received for publication 27 Apr. 2021. Accepted for publication 20 Oct. 2021

Published online 9 December 2021

S.F.H. is the corresponding author. E-mail: sfhutton@ufl.edu.

This is an open access article distributed under the CC BY-NC-ND license (https://creativecommons.org/licenses/by-nc-nd/4.0/). practices to reduce spread of inoculum, can contribute to disease management, especially when combined with soil fumigation (Jones and Overman, 1985; Jones and Woltz, 1968, 1983). However, host resistance is the most effective and economical means of controlling $\mathrm{Fol}$ and has been the primary strategy for controlling $\mathrm{Fol}$ in field-grown tomato production since the 1940s.

There are three races of Fol that infect tomato, and these are distinguished based on their response to three dominant, racespecific resistance genes, each derived from wild tomato species. The $I$ (immunity to fusarium wilt) and $I-2$ genes confer resistance to races 1 (Foll) and 2 (Fol2), respectively. Both genes are located on chromosome 11 and were introgressed from accessions of $S$. pimpinellifolium (Bohn and Tucker, 1939; Paddock, 1950; Simons et al., 1998; Stall and Walter, 1965). I-3, which was introgressed from $S$. pennellii accession LA716, is located on chromosome 7 and confers resistance to race 3 (Fol3) 
and is reported to confer resistance to races 1 and 2 as well (Lim et al., 2008; Scott and Jones, 1989). Currently, only the I, I-2, and $I-3$ genes are used for $\mathrm{Fol}$ resistance in commercial cultivars; but besides these, several other resistance genes have been reported. Sela-Buurlage et al. (2001) described I-4, I-5, and I-6 in $S$. lycopersicum and $S$. pennellii, each conferring Fol2 resistance. More recently, Gonzalez-Cendales et al. (2016) cloned a second dominant Fol3 resistance gene, I-7, from S. pennellii accession LA1724 (PI 414773). Notably, these authors found that the $I-7$ transgene conferred resistance against all three races of $\mathrm{Fol}$, suggesting that I-7 may provide broad efficacy. However, expression of this transgene was driven by the CaMV $35 \mathrm{~S}$ promoter, and the effect of I-7 against other races when under its native promoter has not been clearly demonstrated. Currently, I7 is not widely used for the development of commercial cultivars, likely because $I-3$ is already deployed and provides effective Fol3 control, and also because it was only relatively recently that $I-7$ was distinguished from $I-3$ (Lim et al., 2006).

Fol3 was originally discovered in Australia in 1978 and in Florida in 1982, and it now exists in many production regions around the world (Cai et al., 2003; Correll and Jones, 2014; Grattidge and O'Brien, 1982; Volin and Jones, 1982). Cultivars containing $I-3$ have been commercially available since the early 1990s, but there has been considerable difficulty developing commercially acceptable Fol3-resistant hybrids due to the association of $I-3$ with several negative traits. Some of the earliest Fol3-resistant parental lines showed a higher incidence of blossom end rot than susceptible lines, and this issue is occasionally observed today (Scott 2004). It was also reported that I-3 has a negative effect on fruit size, resulting in a lower percentage of extra-large fruit - the size for which growers usually receive higher prices (Scott, 1999). Furthermore, the $I-3$ locus has been associated with greater sensitivity to bacterial spot disease caused by multiple Xanthomonas species, and Hutton et al. (2014) demonstrated that homozygosity for $I-3$ resulted in up to $20 \%$ more disease severity compared with homozygosity for the susceptible allele. Fortunately, the effects on fruit size and bacterial spot are caused by linkage drag, and reducing the size of the I-3 introgression can eliminate these problems while maintaining Fol3 resistance (Chitwood-Brown et al., 2021; Li et al., 2018).

Such negative effects are not associated with the $I-7$ introgression (Chitwood-Brown et al., 2021) and neither have they been reported for the $I$ or $I-2$ introgressions, which are common resistance genes in much of the commercial germplasm worldwide. In light of this, we hypothesize that problems such as these may be specific to the $I-3$ introgression, and that alternative, novel Fol3 resistance genes might be free of such effects. Furthermore, the discovery and deployment of novel resistance genes may play a critical role in preventing the emergence of new Fol races in the future by expanding the diversity of Fol3 resistance mechanisms. There are more than $40 \mathrm{~S}$. pennellii accessions available from the C.M. Rick Tomato Genetics Resource Center (TGRC) at the University of California, Davis (TGRC, 2011). Scott and Jones (1990) tested many of these and reported broad resistance to multiple Fusarium species in all accessions tested. It is possible that Fol3 resistance in some accessions is based on genetics other than $I-3$ and $I-7$. Considering this, a genetic structure analysis may provide insight into the relationship among these accessions, allowing research efforts to be focused on a smaller number of accessions that are genetically less similar to one another and possibly to retain diversity in resistance alleles. Likewise, such information may be useful for other research efforts seeking to mine S. pennellii for specific traits or novel alleles.

In this study, we investigate a large collection of $S$. pennellii accessions as potential sources of novel Fol3 resistance. We characterize 42 accessions for resistance to all three races of fusarium wilt and identify potential sources of broadly effective resistance alleles. We also evaluate interspecific $\mathrm{F}_{1}$ and backcross populations corresponding to each accession to identify those that are likely sources of novel, Fol3 resistance loci. Finally, we use genotyping by sequencing [GBS (Elshire et al., 2011)] to generate a collection of SNPs between $S$. pennellii accessions and the tomato reference genome for genetic structure analyses.

\section{Materials and Methods}

Plant materials. Seeds of $42 \mathrm{~S}$. pennellii accessions were obtained from TGRC. Each accession was increased by manually sib-mating at least six plants of each accession in a greenhouse located at the University of Florida Gulf Coast Research and Education Center (GCREC) in Wimauma, FL. Information regarding geographic origin for each accession was obtained from the TGRC online database, including latitude, longitude, elevation, and habitat of the collection site, and collection sites were pinpointed on a map (Fig. 1). For each accession, an interspecific $F_{1}$ was made by bulking pollen from six or more plants of the accession and hybridizing with the Fol3 susceptible cultivar, Suncoast (Scott et al., 1985). In the $F_{1}$, pollen was likewise bulked from six or more Fol3-resistant plants and then backcrossed to 'Suncoast' to create modified $\mathrm{BC}_{1} \mathrm{~F}_{1}$ populations for each accession.

Similarly, resistance from accessions LA750, LA1297, LA1299, LA1367, LA1522, LA1734, and LA1926 was advanced into cultivated tomato to the fourth backcross using a modified backcrossing approach, wherein disease assays were again used at each generation to identify resistant plants for backcrossing. Molecular markers linked to I-3 and I-7 (Supplemental Table 1) were used to select away from these known loci and advance novel Fol3 resistance. We selected against the I-3 locus at the $\mathrm{BC}_{1} \mathrm{~F}_{1}$ generation for most accessions (except for LA1926, for which the $I-3$ locus was maintained); and we tested to confirm absence of the $I-7$ locus in $\mathrm{BC}_{4} \mathrm{~F}_{1}$ plants. Screening for $I-7$ was not accomplished sooner because these generations were advanced before the discovery of the chromosomal location of $\mathrm{I}-7$ and the development of genetic resources for this locus by Gonzalez-Cendales et al. (2016). Seed was collected from individual $\mathrm{BC}_{4} \mathrm{~F}_{1}$ plants for each population resulting in segregating $\mathrm{BC}_{4} \mathrm{~F}_{2}$ populations.

Throughout all experiments, seed of accessions, interspecific $\mathrm{F}_{1} \mathrm{~s}$, and $\mathrm{BC}_{1} \mathrm{~F}_{1}$ populations were pretreated in a $2.7 \%$ sodium hypochlorite solution for $30 \mathrm{~min}$ before sowing in spent coal medium (Black Beauty; Harsco Minerals International, Mechanicsburg, PA) in a growth room. Controls used for disease screens included 'Bonny Best' (susceptible to all races), 'Manapal' [Walter et al., 1961 (contains I; resistant to Foll but susceptible to Fol2 and Fol3)], 'Horizon' [Scott et al., 1985 (contains $I$ and I-2; resistant to Foll and Fol2 but susceptible to Fol3)], and Fla. 7547 [Scott and Jones, 1995 (contains I, I-2, and I-3; resistant to Fol1, Fol2, and Fol3)]. 
$\mathbf{A}$

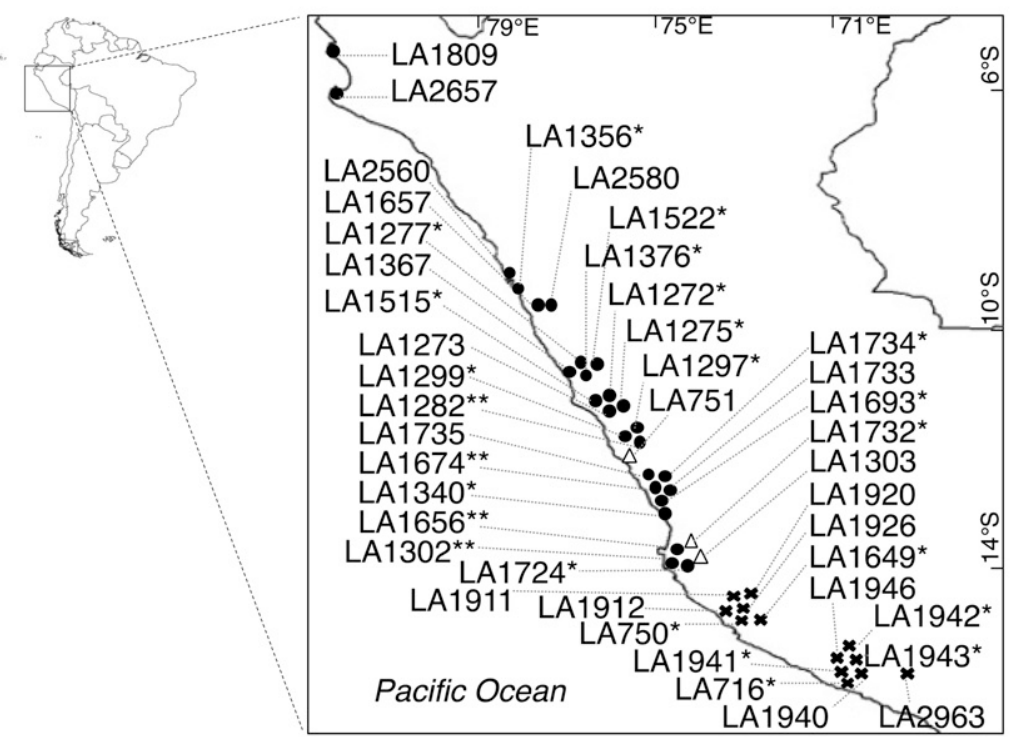

B

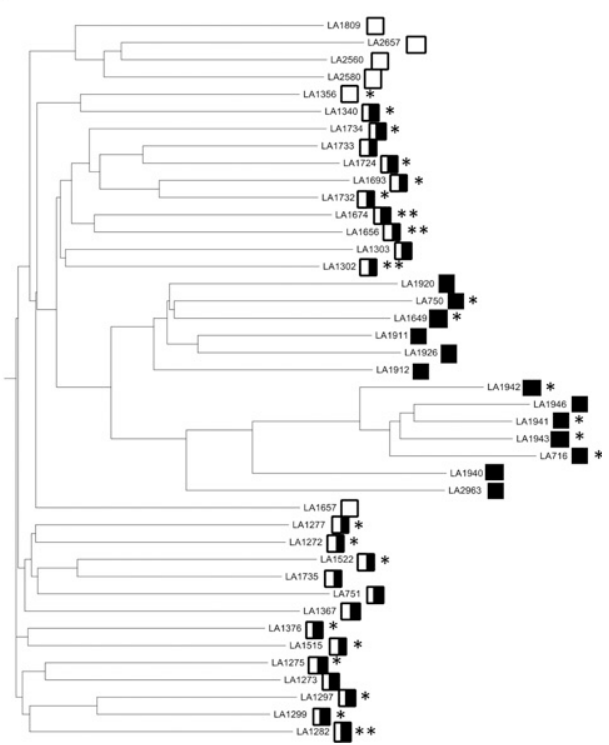

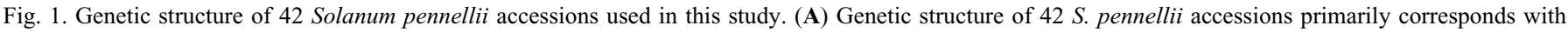
geographic areas. Symbols (circle, empty triangle, cross) are based on three genetic clusters inferred using discriminant analysis of principal components (DAPC) shown on Supplemental Fig. 1. A single or double asterisk indicates the presence of single or multiple novel Fol3 resistance loci, respectively. (B) Neighbor-joining tree of $42 \mathrm{~S}$. pennellii accessions also reveals an association between accessions' geographic origins and phylogenetic clustering. Accessions in the tree are represented by different symbols: empty square (accessions collected north of latitude $10^{\circ} \mathrm{S}$ ); half-filled square (between latitudes $10^{\circ} \mathrm{S}$ and $14^{\circ} \mathrm{S}$ ); and filled square (south of latitude $14^{\circ} \mathrm{S}$ ).

Fusarium WILt disease SCREENING. Fungal strains of Fol races 1 (GEV2997), 2 (GEV3520), and 3 (GEV1400) were obtained from the University of Florida Plant Pathology laboratory at GCREC. The pathogen was grown on potato dextrose agar media at $28^{\circ} \mathrm{C}$ for 1 week before inoculation. Inoculum was prepared by removing the fungal colony with agar from plates and blending into $100-\mathrm{mL}$ deionized water per plate, then adjusting the fungal suspension to a concentration of $10^{7}$ spores $/ \mathrm{mL}$. For each race inoculation, 10- to 14-d-old seedlings were inoculated by dipping the roots into inoculum for $15 \mathrm{~s}$. Inoculated seedlings were immediately transplanted into 128-well trays $\left(38 \mathrm{~cm}^{3}\right.$ cell size; Speedling, Sun City, FL) filled with peatbased soilless media (Speedling) and placed in the greenhouse for disease development. Four to eight plants each of a resistant and a susceptible control were included in each tray; controls included 'Bonny Best' and 'Manapal' for Foll screens, 'Manapal' and 'Horizon' for Fol2 screens, and 'Horizon' and Fla. 7547 for Fol3 screens. Plants were evaluated for disease incidence $\approx 3$ weeks after inoculation according to procedures described previously (Scott et al., 2004). Briefly, plants were rated as diseased if they showed any characteristic external symptoms of fusarium wilt, such as stunting, yellowing of foliage, wilting, enlarged hypocotyl, or death. Stems of plants with questionable symptoms were dissected and rated as diseased if they had vascular browning. Otherwise, plants were scored as healthy and were considered resistant, and numbers of healthy and diseased plants were recorded.

Three separate disease screens were used to evaluate resistance of S. pennellii accessions to Foll, Fol2, and Fol3. Interspecific $\mathrm{F}_{1} \mathrm{~s}$ were evaluated in two separate Fol3 screens, and both $\mathrm{BC}_{1} \mathrm{~F}_{1}$ and $\mathrm{BC}_{4} \mathrm{~F}_{2}$ populations were evaluated in single Fol3 screens. The two screens of interspecific $F_{1}$ s produced similar results, so plant numbers were combined across them. As sufficient plants were available, 32 plants of each accession and each $\mathrm{F}_{1}, 80$ plants of each $\mathrm{BC}_{1} \mathrm{~F}_{1}$, and 56 plants of each $\mathrm{BC}_{4} \mathrm{~F}_{2}$ were evaluated in each disease screen.

Genetic ANALYSIS OF RESISTANCE TO FOL3. Each accession and its interspecific $\mathrm{F}_{1}$ were evaluated for Fol3 resistance to assess the presence/absence of dominant Fol3 resistance alleles. $\mathrm{BC}_{1} \mathrm{~F}_{1}$ families were subsequently evaluated to determine the likelihood that Fol3 resistance is controlled completely or in part by the $I-3$ locus, or by a locus/loci besides $I-3$. Three molecular markers, linked to the $I-3$ locus and distinguishing $S$. pennellii and $S$. lycopersicum alleles, were used for determining the presence of an I-3 locus effect. Before disease development, $\mathrm{BC}_{1} \mathrm{~F}_{1}$ plants were individually screened with one or more of these markers to determine genotype at the $I-3$ locus. For each $\mathrm{BC}_{1} \mathrm{~F}_{1}$ backcross population, each plant was assigned to one of the following four groups: 1) healthy and heterozygous for an introgression at the I-3 locus, 2) healthy and lacking this introgression, 3) diseased and heterozygous for this introgression, or 4) diseased and lacking this introgression.

Within each $\mathrm{BC}_{1} \mathrm{~F}_{1}$ family, a $\chi^{2}$ contingency test was used to determine if the presence of an $S$. pennellii introgression at the $I$ 3 locus affected Fol3 resistance. The null hypothesis for this test was that an introgression at this locus had no effect on resistance, and there would therefore be no difference in the healthy to diseased ratio for plants that possessed and for plants that lacked an I-3 locus introgression. When the null hypothesis was rejected, it was concluded that resistance in the accession is conferred completely or in part by the I-3 locus. Then, where the null hypothesis was accepted, it was concluded that resistance in the accession is not based on $I-3$ but is based entirely on alleles other than $I-3$.

A $\chi^{2}$ test was also used to test for the presence of novel resistance loci. For families of accessions determined to have no 
effect at the I-3 locus, calculations were based on all $\mathrm{BC}_{1} \mathrm{~F}_{1}$ plants; and for families of accessions that were determined to harbor a resistance allele at/near the $I-3$ locus, calculations were based only on plants lacking an $S$. pennellii introgression in this region. Numbers of healthy and diseased plants were tested for goodness of fit to an expected 1:1 and/or 3:1 ratio to test for the presence of one or two alternative resistance loci, respectively. Where families had an unacceptable fit to the 3:1 ratio due to an excess of healthy plants, it was concluded that resistance may be conferred by more than two alternative resistance loci.

Fol3 resistance was further evaluated in $\mathrm{BC}_{4} \mathrm{~F}_{2}$ families, and molecular markers linked to $I-3$ were used as previously described to confirm the presence or absence of this locus. Chisquare analyses of $\mathrm{BC}_{4} \mathrm{~F}_{2}$ families were used to test healthy to diseased ratios for goodness of fit to an expected 3:1 or 15:1 ratio for the presence of one or two resistance genes, respectively.

GenotyPing BY SEQUencing. Young foliar tissue was collected separately from two individual plants from each $S$. pennellii accession. Genomic DNA was extracted using the Plant DNeasy Mini Kit (Qiagen, Hilden, Germany) according to the manufacturer's instructions. The DNA was normalized to $5 \mathrm{ng} \cdot \mu \mathrm{L}^{-1}$ based on quantification with a multimode microplate reader (Synergy 2; Biotek Instruments, Winooki, VT) with the Quant-iT PicoGreen (Thermo Fisher Scientific, Waltham, MA) double-stranded DNA. An ApekI-based GBS library was constructed using previously published protocols, barcode-adapters, and polymerase chain reaction primers (Elshire et al., 2011). The final GBS library (total 84 libraries; two libraries per each accession) was analyzed by automated electrophoresis (2100 Bioanalyzer; Agilent Technologies, Santa Clara, CA) before sequencing to confirm and examine fragment size distribution and quantities. The GBS library was submitted for 101-cycle single-end sequencing on one lane of an Illumina HiSeq 2500 instrument (Illumina, San Diego, CA) at the Weill Cornell Medical College Genomics Resources Core Facility.

One multiplexed sequencing run generated a total of $140,179,147$ reads with barcodes from $42 \mathrm{~S}$. pennellii accessions. The raw sequencing data were processed using the TASSEL 5 GBS v2 pipeline (Bradbury et al., 2007). Bowtie 2 with the -very-sensitive option (Langmead and Salzberg, 2012) was used to align the reads to the tomato reference genome (Tomato SL2.50 ITAG2.4; Sol Genomics Network, 2019). Only 80-base pair kmers (reads) present at least five times in the dataset were considered. Minimum quality score within the barcode and read length was set for 20. SNPs were not filtered by minor allele frequency. For each SNP, only sequence polymorphism common to both individual plant samples of each accession were considered. SNPs with $>95 \%$ missing data were discarded.

GeNETIC STRUCTURE ANALYSES. We explored genetic differentiation among $42 \mathrm{~S}$. pennellii accessions genotyped using the GBS method. We used K-means clustering, which clusters objects to maximize between group variation, for $\mathrm{K}=1$ through 8 implemented in the adegenet package (Jombart, 2008) for the $\mathrm{R}$ software (version 3.0.1; R Foundation, Vienna, Austria). We used the Bayesian information criterion to select the number of clusters that best describes those data. Using the multivariate method [discriminant analysis of principal components (DAPC) (Jombart et al., 2010)], accessions were grouped into three clusters that were genetically diverse from one another. In addition, we built a phylogenetic tree for these accessions using the neighbor-joining method implemented in TASSEL 5 and the same genotype dataset that was used for DAPC.

\section{Results}

Fol Resistance among S. PENnellit aCCessions. Disease screens of 42 accessions confirmed high levels of resistance to Fol in the $S$. pennellii species (Table 1). Overall, these accessions were less resistant to Foll than to Fol2 or Fol3. Whereas 27 accessions demonstrated some level of Foll resistance ( $\geq 50 \%$ healthy plants), only five of these exceeded $90 \%$ healthy plants, whereas 14 accessions had less than 50\% healthy plants. Among the latter, accessions LA1926 (6\% healthy) and LA2657 (15\% healthy) were considered susceptible to Foll. Most accessions were resistant to Fol2, and the percentage of Fol2-resistant plants ranged from $50 \%$ to $90 \%$ among these. LA1926 was the only accession clearly susceptible to Fol2, having 7\% healthy plants. Resistance was highest and most consistent for Fol3, for which 31 accessions had more than $90 \%$ healthy plants, and 39 accessions had more than $70 \%$ healthy plants. LA1733 and LA1912 had less than 70\% healthy plants.

Many of the interspecific $\mathrm{F}_{1} \mathrm{~s}$ were resistant to Fol3 and displayed similar levels of resistance as the parental accession, suggesting dominant gene action and/or homogeneity of resistance alleles within the accession. Some accessions (LA1724, LA1732, LA1809, LA1911, LA1912, LA1926) displayed lower levels of resistance in the interspecific $\mathrm{F}_{1}$, suggesting a lack of dominance and/or segregation of resistance. The possibility of heterogeneity for resistance was suggested for LA1912, as this parental accession LA 1912 had only $63 \%$ healthy plants in the Fol3 assay and $27 \%$ healthy plants in the interspecific $\mathrm{F}_{1}$ (Table 1 ).

To further characterize Fol3 resistance in these materials, $\mathrm{BC}_{1} \mathrm{~F}_{1}$ families corresponding to 40 of the accessions were evaluated in Fol3 disease screens, and molecular markers were used to detect the presence of an introgression at the $I-3$ locus (Table 2 ). Because only two crosses to susceptible cultivated tomato had been made, segregation for an introgression at the $I-3$ locus was expected for all families. However, this locus may or may not confer Fol3 resistance. To identify potential sources of novel resistance loci, we sought first to determine whether the $I-3$ introgression contributed to Fol3 resistance; and second, whether additional resistance loci are likely to exist.

Segregation for Fol3 resistance was observed for each $\mathrm{BC}_{1} \mathrm{~F}_{1}$ family. An introgression near the $I-3$ locus was detected in all populations except for that derived from LA751; here, the linked markers were not polymorphic, and we were therefore unable to test for an I-3 locus effect. Chi-square analysis was used to determine an effect of the $I-3$ locus by comparing the total numbers of healthy and diseased plants with and without an introgression at $I-3$, which indicated the presence of a resistance allele at or near this locus in 18 of the populations (Table 2). For these populations, the presence of alternative resistance loci was determined with $\chi^{2}$ tests using the plants without an introgression at I-3. As expected, an effect at I-3 was detected in LA716, the source of $I-3$ in commercial germplasm, but analysis also indicated the presence of an alternative resistance locus besides I-3 in this accession. The possibility of one additional resistance locus was also indicated for LA750, LA1272, LA1297, LA1299, LA1340, LA1515, LA1522, LA1649, and LA1942. In LA1273, LA1303, LA1735, LA1911, LA1920, LA1926, and LA1946, segregation ratios among plants lacking an introgression at the 


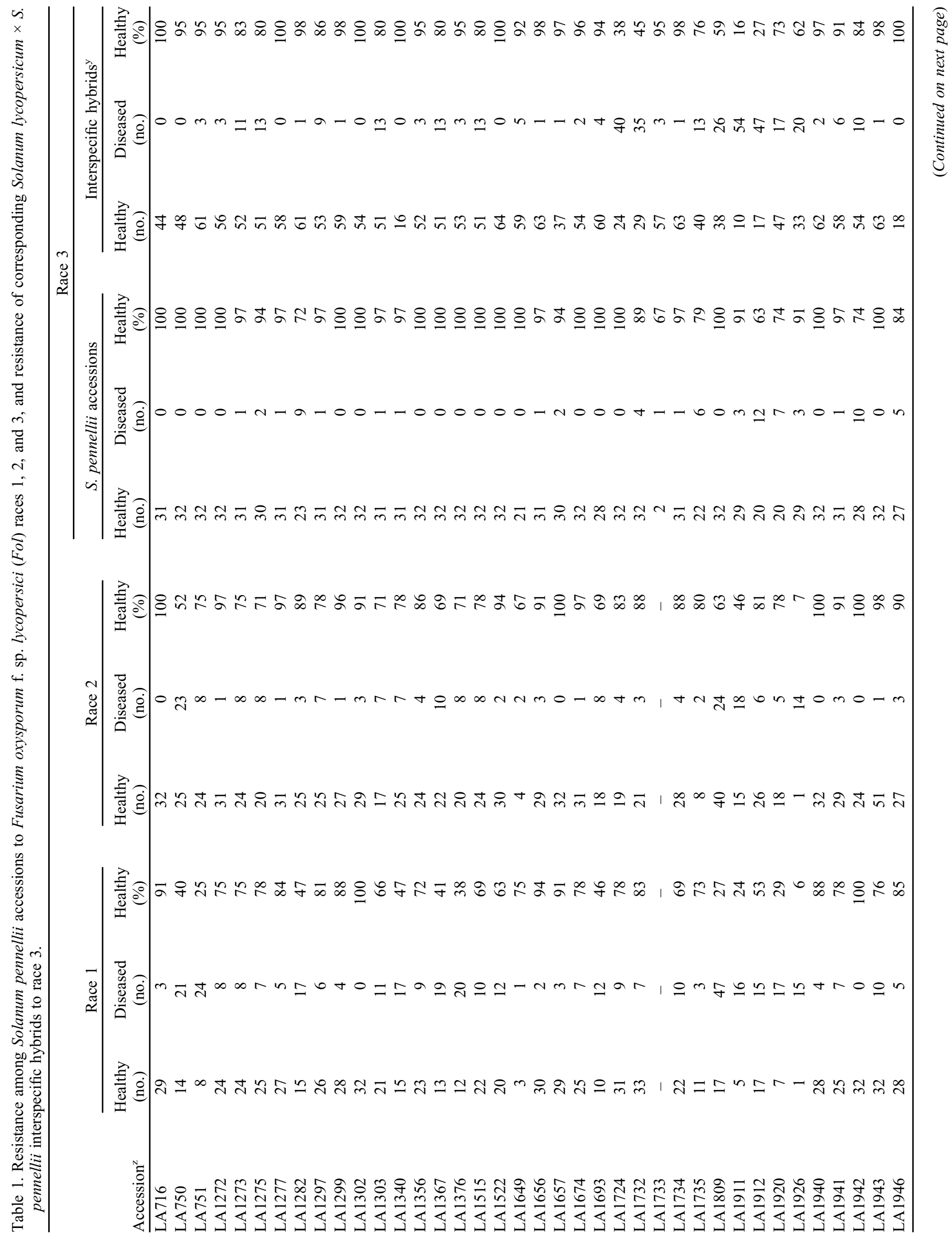




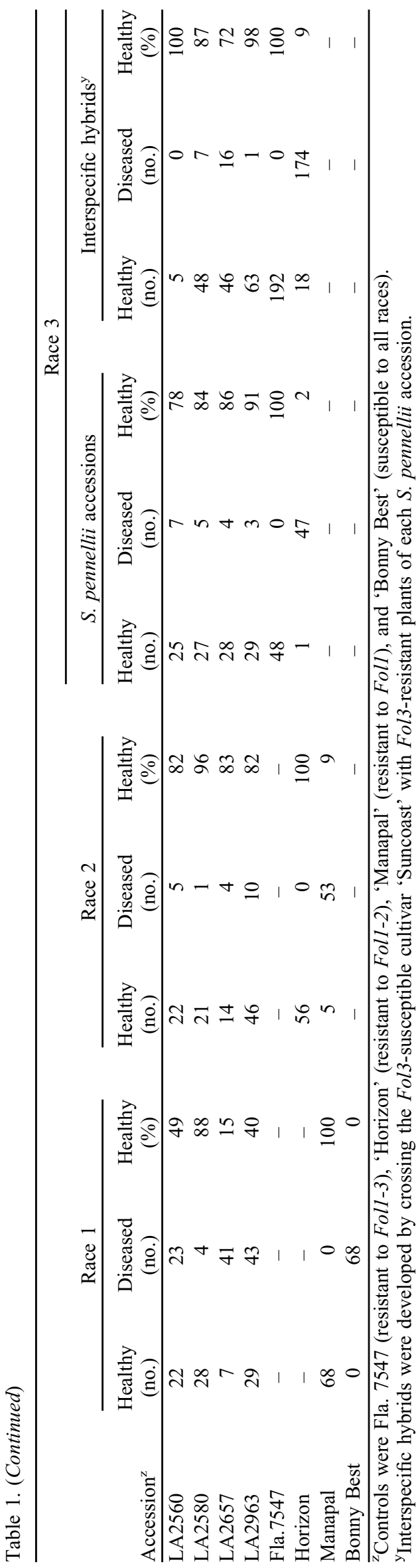

I-3 locus did not support the presence of additional resistance alleles, and these accessions may thus serve little utility toward the identification of novel Fol3 resistance alleles. In LA1302, segregation ratios suggested the possibility of two resistance loci other than $I-3$.

For the 21 populations demonstrating no effect at the $I-3$ locus, the potential number of novel resistance loci were predicted with $\chi^{2}$ tests using the total number of plants screened (Table 2). Based on these analyses, resistance in 10 of the $\mathrm{BC}_{1} \mathrm{~F}_{1}$ families may be conferred by a single locus, while two resistance loci likely confer $\mathrm{Fol3}$ resistance in accessions LA1282, LA1656, and LA1674.

Besides those mentioned previously, eight $\mathrm{BC}_{1} \mathrm{~F}_{1}$ families fit none of the segregation ratios tested, primarily because of a deficiency of resistant plants (Table 2). LA1912 was among these and demonstrated the lowest levels of resistance of all $\mathrm{BC}_{1} \mathrm{~F}_{1}$ families. LA1367 was also among these, but in the case of this accession, there was no deficiency of resistant plants. Rather, its 51:29 ratio of healthy:diseased plants was slightly skewed such that it fit neither a 1:1 nor a 3:1 segregation ratio. Thus, although $\chi^{2}$ analyses were not conclusive, there is evidence that LA1367 contains at least one alternative Fol3 resistance locus.

We continued to advance resistance from some accessions into cultivated tomato by selecting resistant plants from disease assays for backcrossing. Resistance to Fol3 in several $\mathrm{BC}_{4} \mathrm{~F}_{2}$ families is shown in Table 3. Chi-square tests of segregation ratios were used to substantiate predictions made in the $\mathrm{BC}_{1} \mathrm{~F}_{1}$ families. We also used molecular markers linked to $I-3$ to confirm absence of an introgression (or in the case of LA1926, genotype for each $\mathrm{BC}_{4} \mathrm{~F}_{2}$ plant) at this locus in each family. LA750, LA1297, LA1299, and LA1522 were previously determined to have an effect at the $I-3$ locus in addition to one alternative resistance locus. Here, $I-3-$ linked markers confirmed the absence of an $I-3$ introgression in these $\mathrm{BC}_{4} \mathrm{~F}_{2}$ families, and segregation ratios further supported the presence of one alternative locus conferring resistance in each family. As mentioned previously, the presence of at least one alternative Fol3 resistance locus was evidenced, although segregation ratios in its $\mathrm{BC}_{1} \mathrm{~F}_{1}$ family did not fit either a 1:1 or 3:1 ratio. However, $\chi^{2}$ analyses of the $\mathrm{BC}_{4} \mathrm{~F}_{2}$ population for LA1367 indicated two alternative genes conferring resistance (Table 3). LA1734 was formerly determined to have no effect at the I-3 locus and to contain one alternative resistance locus; analyses confirmed the absence of any introgression at the $I-3$ locus and supported the presence of a single novel locus conferring resistance. Resistance from LA1926, which was previously determined to be conferred solely by the $I-3$ locus, was also advanced, and the segregating $\mathrm{BC}_{4} \mathrm{~F}_{2}$ population was subjected to disease and marker screens, as previously described. Chi-square analyses confirmed segregation of a single resistance locus, and further testing verified that the $I-3$ locus in fact cosegregated with resistance in this line $(P<0.0001)$.

GeNeTiC STRUCtURe aNALYSES fOR $\boldsymbol{S}$. PENNELLII ACCESSIONS. In this study, all accessions originated from Peru (Fig. 1A). Their geographic collection sites are distributed along the entire west coast, with the greatest distance between collection sites being $1537 \mathrm{~km}$ between accessions LA1089 and LA2963. The smallest distance between collection sites was less than $1 \mathrm{~km}$, and this was observed between LA751 and LA1282, between LA1911 and LA1912, and between LA1942 and LA1943. 
Table 2. Genetic analysis of Solanum lycopersicum $\times$ Solanum pennellii $\mathrm{BC}_{1} \mathrm{~F}_{1}$ populations for identifying potential sources of novel resistance to fusarium wilt race 3 .

\begin{tabular}{|c|c|c|c|c|c|c|c|c|c|}
\hline \multirow[b]{3}{*}{$\begin{array}{l}\mathrm{BC}_{1} \mathrm{~F}_{1} \\
\text { population }\end{array}$} & \multicolumn{6}{|c|}{$I-3$ locus introgression } & \multicolumn{3}{|c|}{ Alternative resistance loci } \\
\hline & \multicolumn{2}{|c|}{ Heterozygous } & \multicolumn{2}{|c|}{ Absent } & \multicolumn{2}{|c|}{ Locus effect } & \multicolumn{2}{|c|}{$\chi^{2}$ probability $^{x}$} & \multirow[b]{2}{*}{$\begin{array}{l}\text { Loci } \\
\text { (no.) }\end{array}$} \\
\hline & $\begin{array}{c}\text { Healthy } \\
\text { (no.) }\end{array}$ & $\begin{array}{c}\text { Diseased } \\
\text { (no.) }\end{array}$ & $\begin{array}{c}\text { Healthy } \\
\text { (no.) }\end{array}$ & $\begin{array}{l}\text { Diseased } \\
\text { (no.) }\end{array}$ & $\chi^{2}$ probability & Conclusion $^{\mathrm{y}}$ & $(1: 1)$ & $(3: 1)$ & \\
\hline LA716 & 37 & 4 & 17 & 20 & 0.000 & Yes & 0.622 & 0.000 & 1 \\
\hline LA750 & 32 & 5 & 14 & 24 & 0.000 & Yes & 0.105 & 0.000 & 1 \\
\hline LA751 & 0 & 0 & 20 & 40 & - & - & 0.010 & 0.000 & 0 \\
\hline LA1272 & 39 & 5 & 11 & 18 & 0.000 & Yes & 0.194 & 0.000 & 1 \\
\hline LA1273 & 22 & 14 & 6 & 16 & 0.012 & Yes & 0.033 & 0.000 & 0 \\
\hline LA1275 & 14 & 19 & 20 & 26 & 0.926 & No & 0.216 & 0.000 & 1 \\
\hline LA1277 & 21 & 17 & 10 & 18 & 0.116 & No & 0.622 & 0.000 & 1 \\
\hline LA1282 & 35 & 14 & 16 & 13 & 0.145 & No & 0.007 & 0.050 & 2 \\
\hline LA1297 & 28 & 11 & 14 & 23 & 0.003 & Yes & 0.139 & 0.000 & 1 \\
\hline LA1299 & 20 & 0 & 10 & 10 & 0.000 & Yes & 1.000 & 0.010 & 1 \\
\hline LA1302 & 15 & 0 & 11 & 4 & 0.032 & Yes & 0.071 & 0.881 & 2 \\
\hline LA1303 & 15 & 11 & 11 & 28 & 0.017 & Yes & 0.006 & 0.000 & 0 \\
\hline LA1340 & 23 & 13 & 16 & 23 & 0.048 & Yes & 0.262 & 0.000 & 1 \\
\hline LA1356 & 30 & 17 & 12 & 16 & 0.077 & No & 0.299 & 0.000 & 1 \\
\hline LA1367 & 11 & 3 & 40 & 26 & 0.204 & No & 0.014 & 0.020 & $-{ }^{\mathrm{w}}$ \\
\hline LA1376 & 20 & 11 & 19 & 17 & 0.331 & No & 0.179 & 0.002 & 1 \\
\hline LA1515 & 22 & 5 & 27 & 26 & 0.008 & Yes & 0.891 & 0.000 & 1 \\
\hline LA1522 & 27 & 9 & 16 & 28 & 0.001 & Yes & 0.070 & 0.000 & 1 \\
\hline LA1649 & 38 & 9 & 12 & 18 & 0.000 & Yes & 0.273 & 0.000 & 1 \\
\hline LA1656 & 22 & 11 & 18 & 10 & 0.845 & No & 0.015 & 0.089 & 2 \\
\hline LA1674 & 34 & 14 & 19 & 13 & 0.288 & No & 0.004 & 0.071 & 2 \\
\hline LA1693 & 13 & 8 & 16 & 21 & 0.172 & No & 1.000 & 0.000 & 1 \\
\hline LA1724 & 11 & 15 & 20 & 19 & 0.478 & No & 0.710 & 0.000 & 1 \\
\hline LA1732 & 9 & 10 & 12 & 18 & 0.612 & No & 0.317 & 0.000 & 1 \\
\hline LA1733 & 10 & 16 & 11 & 26 & 0.469 & No & 0.008 & 0.000 & 0 \\
\hline LA1734 & 7 & 6 & 11 & 16 & 0.435 & No & 0.527 & 0.000 & 1 \\
\hline LA1735 & 9 & 14 & 9 & 43 & 0.041 & Yes & 0.000 & 0.000 & 0 \\
\hline LA1809 & 10 & 17 & 10 & 32 & 0.237 & No & 0.000 & 0.000 & 0 \\
\hline LA1911 & 7 & 6 & 6 & 22 & 0.038 & Yes & 0.002 & 0.000 & 0 \\
\hline LA1912 & 3 & 34 & 2 & 29 & 0.794 & No & 0.000 & 0.000 & 0 \\
\hline LA1920 & 20 & 17 & 8 & 34 & 0.001 & Yes & 0.000 & 0.000 & 0 \\
\hline LA1926 & 12 & 6 & 1 & 16 & 0.000 & Yes & 0.000 & 0.000 & 0 \\
\hline LA1940 & 15 & 20 & 11 & 35 & 0.070 & No & 0.001 & 0.000 & 0 \\
\hline LA1941 & 20 & 19 & 21 & 20 & 0.996 & No & 0.823 & 0.000 & 1 \\
\hline LA1942 & 25 & 9 & 21 & 20 & 0.048 & Yes & 0.876 & 0.000 & 1 \\
\hline LA1943 & 23 & 16 & 23 & 15 & 0.890 & No & 0.087 & 0.002 & 1 \\
\hline LA1946 & 10 & 10 & 8 & 33 & 0.014 & Yes & 0.000 & 0.000 & 0 \\
\hline LA2580 & 4 & 11 & 20 & 33 & 0.428 & No & 0.015 & 0.000 & 0 \\
\hline LA2657 & 13 & 17 & 9 & 32 & 0.054 & No & 0.001 & 0.000 & 0 \\
\hline LA2963 & 7 & 29 & 6 & 33 & 0.643 & No & 0.000 & 0.000 & 0 \\
\hline
\end{tabular}

${ }^{\mathrm{z}}$ Backcross populations were developed from crossing fusarium wilt race 3-resistant plants from each S. pennellii accession and from each subsequent interspecific hybrid with the race 3-susceptible cultivar Suncoast.

${ }^{y}$ An effect at the $I-3$ locus was determined using $\chi^{2}$ contingency tests in which the null hypothesis was that no difference was observed in the ratio of healthy to diseased plants whether the $I-3$ locus was present or absent. "No" indicates that the null was accepted and there was no effect of the $I-3$ locus, and "yes" indicates that the null was rejected and the locus was determined to have an effect.

${ }^{\mathrm{x}}$ Chi-square tests were conducted using the total number of plants when an $I-3$ effect was absent in an $\mathrm{BC}_{1} \mathrm{~F}_{1}$ population; and tests were conducted using only $i-3 / i-3$ plants when an $I-3$ effect was present in an $\mathrm{BC}_{1} \mathrm{~F}_{1}$ population $(P<0.05)$. The analysis tested whether segregation fit a $1: 1$ or $3: 1$ (healthy to diseased) ratio, indicating the presence of one or two resistance loci, respectively.

${ }^{\mathrm{w}}$ Although the number of healthy and diseased plants for the population with resistance derived from LA1367 fit neither a 1:1 nor a 3:1 segregation ratio, the number of healthy plants is evidence that at least one Fol3 resistance locus is present.

Analysis of data from GBS identified 1,378,871 SNPs across all accessions (Supplemental Tables 2 and 3). The number of monoallelic SNPs per accession ranged from 24,157 to 33,735 , and the number of heterozygous SNPs per accession ranged from 3005 to 7205 . The proportion of heterozygous SNPs was high, ranging from $10 \%$ to $27 \%$ across accessions and averaging $20 \%$. This was likely due to the outcrossing nature of the species, and for this reason, heterozygous SNPs 
Table 3. Analysis of advanced resistance to fusarium wilt race 3 in Solanum lycopersicum $\mathrm{BC}_{4} \mathrm{~F}_{2}$ populations.

\begin{tabular}{lcccc}
\hline \multirow{2}{*}{$\begin{array}{l}\text { Resistance } \\
\text { derived from }\end{array}$} & $\begin{array}{c}\text { Healthy } \\
\text { (no.) }\end{array}$ & $\begin{array}{c}\text { Diseased } \\
\text { (no.) }\end{array}$ & $(3: 1)$ & \multicolumn{2}{c}{$\chi^{2}$ probability $^{z}$} \\
\hline LA750 & 45 & 11 & 0.355 & 0.000 \\
LA1297 & 36 & 20 & 0.064 & 0.000 \\
LA1299 & 36 & 20 & 0.064 & 0.000 \\
LA1367 & 49 & 7 & 0.031 & 0.053 \\
LA1522 & 43 & 13 & 0.758 & 0.000 \\
LA1734 & 47 & 9 & 0.123 & 0.002 \\
LA1926 & 42 & 10 & 0.337 & 0.000 \\
LA750 & 45 & 11 & 0.355 & 0.000 \\
\hline
\end{tabular}

${ }^{\mathrm{z}}$ Chi-square analyses were conducted to compare the segregation ratios of healthy and diseased plants to the ratios expected when one $(3: 1)$ or two $(15: 1)$ resistance genes are present.

were not excluded from further analyses. Upon comparing all SNP loci among the accessions, a final set of 49,120 unique SNP loci were determined, and these were used for subsequent analyses.

K-means clustering indicated that different patterns of genetic structure could be used to describe the genotype data of all 42 accessions (Supplemental Fig. 1). The mode of $\mathrm{K}=3$ found significant structuring between accessions collected from areas above an approximate latitude $14^{\circ} \mathrm{S}$ (two clusters with 29 accessions) and accessions collected from below this latitude (a single cluster with 13 accessions) (Fig. 1A). A third cluster with three accessions (LA751, LA1732, LA1303) showed no clear geographic differentiation from the rest of the accessions collected from areas above an approximate latitude $14^{\circ} \mathrm{S}$, but was reassigned to a different genetic structure.

We also investigated genetic structure using a phylogenetic analysis, and this likewise revealed an association between geographic origins and the occurrence of phylogenetic clustering (Fig. 1B). Importantly, we observed that the accessions with novel Fol3 resistance alleles are not confined to a single clade but are present in various clades. Among all accessions, the smallest pairwise genetic distance was between accessions LA716 and LA1943, and the largest genetic distance was between LA1943 and LA2560.

\section{Discussion}

Much of the disease resistance used in hybrid tomato breeding is conferred by dominant resistance genes. The present study investigated a collection of $42 \mathrm{~S}$. pennellii accessions for their potential to harbor novel Fol3 resistance loci. The use of screenings at the $\mathrm{F}_{1}, \mathrm{BC}_{1} \mathrm{~F}_{1}$, and subsequent $\mathrm{BCF}_{1}$ generations has likely biased our results toward detection of dominant resistance alleles that confer a high level of resistance. We used $\chi^{2}$ analyses to test segregation ratios in backcross families to determine which accessions are likely to contain novel Fol3 resistance loci, thereby providing some direction for programs that may wish to pursue research in this area. Although these estimates can be useful, they may not be completely accurate in all cases, because segregation ratios in wide crosses such as ours can deviate from normal (Rick, 1969). The estimates were also based on the assumption that all detectable resistance alleles were homogeneously present in the interspecific $F_{1}$. Although we used only Fol3-resistant $\mathrm{F}_{1}$ plants to generate $\mathrm{BC}_{1} \mathrm{~F}_{1}$ seed, we recognize that $S$. pennellii is primarily an outcrossing species, and that this assumption may have been violated in some instances if multiple resistance alleles were present and segregating within an accession.

Our screening confirmed a very high level of Fol3 resistance in most S. pennellii accessions. Resistance to Fol3 appeared to be dominant in most accessions, as evidenced by the high survival rates among interspecific $F_{1} s$. This is of particular practical importance, given the utility of dominant resistance genes for hybrid breeding. In general, survival rates in the $F_{1} \mathrm{~s}$ were slightly lower relative to the donor accessions, which might indicate some level of incomplete dominance. But another likely explanation is that this resulted from heterogeneity of resistance, which may also explain why Fol3 resistance deviated from $100 \%$ for several of the accessions. $F_{1}$ survival rates for some of the accessions (i.e., LA1724, LA1732, LA1911, and LA1912) were markedly low, possibly due to heterogeneity and/or heterozygosity of resistance within the accessions, incomplete dominance of resistance, or segregation distortion for the resistance alleles. In the analysis of $\mathrm{BC}_{1} \mathrm{~F}_{1}$ populations, both LA1724 and LA1732 were predicted to contain one resistance locus. LA1724 is the reported source of Fol3 resistance gene $\mathrm{I}-7$, and it is likely that the resistance in this population is due to this gene. Furthermore, Catanzariti et al. (2015) demonstrated that I-7 confers resistance to Foll and Fol2, and we also observed LA1724 to have resistance to Foll (78\% healthy) and Fol2 (83\% healthy) (Table 1). Therefore, for the purpose of identifying novel sources of $\mathrm{Fol}$ resistance, this accession may be of minimal interest.

Several accessions displayed lower resistance or were susceptible to Foll and/or Fol2. These accessions may therefore be less desirable as potential sources of novel alleles, if the goal is to identify sources of broad resistance toward improved durability. Bournival et al. (1990) suggested that the $I-3$ introgression confers resistance to Fol3 as well as Foll and Fol2, but it was unclear from this work whether resistances to races 1 and 2 were conferred by I-3 or by linked genes. Although Scott et al. (2004) and Sarfatti et al. (1991) reported that Foll resistance in LA716 is conferred by the $I-1$ gene located near $I-3$, findings of Do et al. (2016) rather suggest that $I-3$ alone confers at least partial resistance to Foll in addition to resistance to races 2 and 3. Despite the lack of continuity among these studies, the evidence that $I-3$ provides at least partial resistance to multiple races supports that $I-3$ should be pyramided with, rather than replaced by, any novel Fol3 resistance alleles that may later be discovered, especially if these do not provide comparable disease control to Fol races 1 to 3 as $I-3$. Our findings demonstrate that resistance to all three $\mathrm{Fol}$ races is present in multiple accessions, but further work will be needed to determine if a specific novel Fol3 resistance locus is likewise effective against races 1 and 2.

Whereas Fol3 resistance cosegregated with the I-3 locus in some backcross populations, 23 accessions are expected to contain one or more alternative resistance loci. Seven of these (i.e., LA750, LA1282, LA1340, LA1376, LA1522, LA1649, and LA1693) had questionable Foll and/or Fol2 resistance and thus may not be first choices in pursuit of novel, durable Fol3 resistance genes. The remaining 16 accessions (i.e., LA716, LA1272, LA1275, LA1277, LA1297, LA1299, LA1302, LA1356, LA1515, LA1656, LA1674, LA1732, LA1734, LA1941, LA1942, and LA1943) are all strong candidate sources for novel Fol3 resistance genes and also demonstrated high levels of Foll and Fol2 resistance. For each of these accessions, $F o l 3$ resistant $\mathrm{BC}_{1} \mathrm{~F}_{1}$ plants that 
lacked an I-3 locus introgression were used for further backcrossing, and resistance was advanced to the fourth backcross. Segregation in $\mathrm{BC}_{4} \mathrm{~F}_{2}$ families for several of these sources also supported the alternative locus predictions in the $\mathrm{BC}_{1} \mathrm{~F}_{1}$. One alternative resistance locus likely confers resistance in $\mathrm{BC}_{4} \mathrm{~F}_{2}$ families of LA750, LA1297, LA1299, LA1522, and LA1734; while LA1367 could contribute two novel resistance genes.

Because of our intentional selection efforts against known Fol3 resistance loci, it is not expected that any of the advanced backcross populations generated through this work will contain $I-3, I-7$, or alternative alleles at these loci. However, it is possible that other reported $\mathrm{Fol}$ resistance loci may be responsible for the resistance responses that were observed. Sela-Buurlage et al. (2001) identified I-5 and I-6 from S. pennellii accession LA716, each of which conferred resistance to Fol2. The authors also identified regions on chromosome 11 that were associated with resistances to races 1 and 2 , concluding that these regions may contain $S$. pennellii alleles of the $I$ and/or $I$ - 2 genes from $S$. pimpinellifolium. Further research is under way to determine the locations of alternative resistance loci in several accessions and to verify whether resistance is conferred by novel or by previously reported loci. In their study, Sela-Buurlage et al. (2001) reported the locus syntenic with the $I$ gene to confer almost complete resistance to Fol2. This suggests the possibility that some alternative alleles may confer resistance to multiple races of fusarium wilt, increasing their utility toward developing more durable resistance to this pathogen.

Data from GBS was successfully used to identify SNPs and to genotype all $42 \mathrm{~S}$. pennellii accessions used in this study. This information may provide a valuable resource for researchers wishing to use the $S$. pennellii species in their programs; for example, sequence polymorphisms identified in diverse sources could be used to capture existing genetic diversity and develop molecular markers linked to resistance in each specific accession or to obtain marker saturation near the target gene(s) region in mapping populations.

In general, the genetic structure of $S$. pennellii elucidated from GBS data evidences the divergence of the southern accessions from the northern accessions, corresponding with geographic areas that $\operatorname{span} \approx 10^{\circ}$ latitude. However, no clear geographic pattern was evident with regard to presence of alternative Fol3 resistance loci. In addition, genetic structure did not primarily correspond to the observed alternative loci. The range in genetic distance among all accessions was relatively small, which resulted from the fact that all accessions belong to the same species. Nonetheless, the distinguishable genetic structure among accessions means that the GBS data can potentially be used to classify accessions based on their similarity with one another. Thus, whether investigating novel Fol3 resistance loci or other $S$. pennellii traits, researchers may choose to work with one or more accessions from each of the two major genetic clusters (viz. the circle and the cross symbols in Fig. 1) to maximize the genetic diversity represented in their populations. Likewise, the highly significant correlation between genetic distance and location of origin that was observed suggests that one could accomplish the same goal by arbitrarily choosing accessions from the northern and southern geographical collection sites. With regard to Fol3 resistance, our hypothesis is that accessions that are least related to one another may be less likely to contain the same alternative resistance gene to Fol3, and this also could be investigated with further research.
Despite the adoption of cultural practices that improve management of fusarium wilt in tomato, the utilization of Folresistant cultivars has been the primary strategy for disease control since 1939. Cultivar resistance is primarily based on three single, dominant resistance genes: $I, I-2$, and $I-3$. Considering the history of R genes in many crops, together with the fact that both $I$ and $I$ 2 were overcome with time, it is almost certain that $I-3$ will eventually be overcome by the emergence of a new Fol strain(s). However, the introduction of additional $F o l$ resistance genes for pyramiding with those currently being used is likely to improve the durability of resistance. As an example, the $I-3$ and $I-7$ genes belong to different gene families and also differ in their mechanisms of resistance to Fol3, and these genes are already being pyramided in some breeding programs (Catanzariti et al., 2015; Gonzalez-Cendales et al., 2016; Lim et al., 2006). Similarly, sources of novel Fol3 resistance loci identified through the present study and currently being pursued may help to expand the Fol resistance gene resources that are available toward this end.

\section{Literature Cited}

Bradbury, P.J., Z. Zhang, D.E. Kroon, T.M. Casstevens, Y. Ramdoss, and E.S. Buckler. 2007. TASSEL: Software for association mapping of complex traits in diverse samples. Bioinformatics 23:2633-2635, https://doi.org/10.1093/bioinformatics/btm308.

Bohn, G.W. and C.M. Tucker. 1939. Immunity to fusarium wilt in the tomato. Science 89:603-604.

Bournival, B.L., C.E. Vallejos, and J.W. Scott. 1990. Genetic analysis of resistances to races 1 and 2 of Fusarium oxysporum f. sp. lycopersici from the wild tomato Lycopersicon pennellii. Theor. Appl. Genet. 79:641-645, https://doi.org/10.1007/BF00226877.

Cai, G., L.R. Gale, R.W. Schneider, H.C. Kistler, R.M. Davis, K.S. Elias, and E.M. Miyao. 2003. Origin of race 3 of Fusarium oxysporum f. sp. lycopersici at a single site in California. Phytopathology 93:1014-1022, https://doi.org/10.1094/PHYTO.2003.93.8.1014.

Catanzariti, A.M., G.T. Lim, and D.A. Jones. 2015. The tomato I-3 gene: A novel gene for resistance to fusarium wilt disease. New Phytol. 207:106-118, https://doi.org/10.1111/nph.13348.

Chitwood-Brown, J., G.E. Vallad, T.G. Lee, and S.F. Hutton. 2021. Characterization and elimination of linkage-drag associated with fusarium wilt race 3 resistance genes. Theor. Appl. Genet. 134:2129-2140, https://doi.org/10.1007/s00122-021-03810-5.

Correll, J. and J.P. Jones. 2014. Fusarium wilt, p. 28-29. In: J.B. Jones, T.A. Zitter, T.M. Momol, and S.A. Miller (eds.). Compendium of tomato diseases and pests, 2nd ed. Amer. Phytopathol. Soc., St. Paul, MN.

Do, T.T.H., A.M. Catanzariti, G.T.T. Lim, and D.A. Jones. 2016. Evidence against the existence of genes for resistance to Fusarium oxysporum f. sp. lycopersici races 1 and 2 on Solanum pennellii chromosome 7 additional to I-3. Acta Hort. 1207:19-26, https://doi. org/10.17660/ActaHortic.2018.1207.2.

Elshire, R.J., J.C. Glaubitz, Q. Sun, J.A. Poland, K. Kawamoto, E.S. Buckler, and S.E. Mitchell. 2011. A robust, simple genotyping-bysequencing (GBS) approach for high diversity species. PLoS One 6:e19379, https://doi.org/10.1371/journal.pone.0019379.

Gonzalez-Cendales, Y., A.M. Catanzariti, B. Baker, D.J. McGrath, and D.A. Jones. 2016. Identification of I-7 expands the repertoire of genes for resistance to fusarium wilt in tomato to three resistance gene classes. Mol. Plant Pathol. 17:448-463, https://doi.org/10.1111/ mpp. 12294.

Grattidge, R. and R.G. O'Brien. 1982. Occurrence of a third race of fusarium wilt of tomatoes in Queensland. Plant Dis. 66:165-166, https://doi.org/10.1094/PD-66-165.

Hutton, S.F., J.W. Scott, and G.E. Vallad. 2014. Association of fusarium wilt race 3 resistance gene, $I-3$, on chromosome 7 with increased susceptibility to bacterial spot race T4 in tomato. J. Amer. Soc. Hort. Sci. 139:282-289, https://doi.org/10.21273/JASHS.139.3.282. 
Jombart, T. 2008. Adegenet: A R package for the multivariate analysis of genetic markers. Bioinformatics 24:1403-1405, https://doi.org/ 10.1093/bioinformatics/btn129.

Jombart, T., S. Devillard, and F. Balloux. 2010. Discriminant analysis of principal components: A new method for the analysis of genetically structured populations. BMC Genet. 11:94, https://doi.org/10.1186/1471-2156-11-94.

Jones, J.P. and A.J. Overman. 1985. Management of fusarium wilt, fusarium crown rot, verticillium wilt (race 2), southern blight, and root-knot of tomato on fine sandy soils. Proc. Florida State Hort. Soc. 98:225-231.

Jones, J.P. and S.S. Woltz. 1968. Field control of fusarium wilt (race 2) of tomato by liming and stake disinfestation. Proc. Florida State Hort. Soc. 81:187-191.

Jones, J.P. and S.S. Woltz. 1983. Cultural control of fusarium wilt race 3 of tomato. Proc. Florida State Hort. Soc. 96:82-83.

Langmead B. and S.L. Salzberg. 2012. Fast gapped-read alignment with Bowtie 2. Nat. Methods 9:357-359, https://doi.org/10.1038/nmeth.1923.

Li, J., J. Chitwood, N. Menda, L. Mueller, and S.F. Hutton. 2018. Linkage between the $I-3$ gene for resistance to fusarium wilt race 3 and increased sensitivity to bacterial spot in tomato. Theor. Appl. Genet. 131:145-155, https://doi.org/10.1007/s00122-017-2991-4.

Lim, G.T.T., G.P. Wang, M.N. Hemming, S. Basuki, D.J. McGrath, B.J. Carroll, and D.A. Jones. 2006. Mapping the $I-3$ gene for resistance to fusarium wilt in tomato: Application of an I-3 marker in tomato improvement and progress towards the cloning of $I-3$. Australas. Plant Pathol. 35:671-680, https://doi.org/10.1071/AP06073.

Lim, G.T.T., G.P. Wang, M.N. Hemming, D.J. McGrath, and D.A. Jones. 2008. High resolution genetic and physical mapping of the $I-3$ region of tomato chromosome 7 reveals almost continuous microsynteny with grape chromosome 12 but interspersed microsynteny with duplication on Arabidopsis chromosomes 1, 2 and 3. Theor. Appl. Genet. 118:57-75, https://doi.org/10.1007/s00122-008-0876-2.

McGrath, D.J., D. Gillespie, and L. Vawdrey. 1987. Inheritance of resistance to Fusarium oxysporum f. sp. lycopersici races 2 and 3 in Lycopersicon pennellii. Aust. J. Agr. Res. 38:729-733, https://doi. org/10.1071/AR9870729.

Paddock, E.F. 1950. A tentative assignment of fusarium-immunity locus to linkage group 5 in tomato. Genetics 35:683-684.

Rick, C.M. 1969. Controlled introgression of chromosomes of Solanum pennellii into Lycopersicon esculentum: Segregation and recombination. Genetics 62:753-768.

Sarfatti, M., M. Abu-Abied, J. Katan, and D. Zamir. 1991. RFLP mapping of I1, a new locus in tomato conferring resistance against Fusarium oxysporum f. sp. lycopersici race 1. Theor. Appl. Genet. 82:22-26, https://doi.org/10.1007/BF00231273.
Scott, J.W. 1999. Tomato plants heterozygous for fusarium wilt race 3 resistance develop larger fruit than homozygous resistant plants. Proc. Florida State Hort. Soc. 112:305-307.

Scott, J.W. 2004. Fla. 7946 tomato breeding line resistant to Fusarium oxysporum f.sp. lycopersici races 1, 2, and 3. HortScience 39:440-441, https://doi.org/10.21273/HORTSCI.39.2.440.

Scott, J.W. and J.P. Jones. 1989. Monogenic resistance in tomato to Fusarium oxysporum f.sp. lycopersici race 3. Euphytica 40:49-53, https://doi.org/10.1007/BF00023296.

Scott, J.W. and J.P. Jones. 1990. Soil-borne fungal resistance in Lycopersicon pennellii accessions. HortScience 25:1068 (abstr.).

Scott, J.W. and J.P. Jones. 1995. Fla. 7547 and Fla. 7481 tomato breeding lines resistant to Fusarium oxysporum f. sp. lycopersici races 1, 2 and 3. HortScience 30:645-646, https://doi.org/10.21273/ HORTSCI.30.3.645.

Scott, J.W., J.A. Bartz, H.H. Bryan, P.H. Everett, D.D. Gull, T.K. Howe, P.J. Stoffella, and R.B. Volin. 1985. Horizon, a fresh market tomato with concentrated fruit set. Univ. Florida Agr. Exp. Sta. Circ. S-323.

Scott, J.W., H.A. Agrama, and J.P. Jones. 2004. RFLP-based analysis of recombination among resistance genes to fusarium wilt races 1,2 and 3 in tomato. J. Amer. Soc. Hort. Sci. 129:394-400, https://doi. org/10.21273/JASHS.129.3.0394.

Sela-Buurlage, M.B., O. Budai-Hadrian, Q. Pan, L. Carmel-Goren, R. Vunsch, D. Zamir, and R. Fluhr. 2001. Genome-wide dissection of Fusarium resistance in tomato reveals multiple complex loci. Mol. Genet. Genomics 265:1104-1111, https://doi.org/10.1007/s004380100509.

Simons, G., J. Groenendijk, J. Wijbrandi, M. Reijans, J. Groenen, P. Diergaarde, T. Van der Lee, M. Bleeker, J. Onstenk, M. de Both, M. Haring, J. Mes, B. Cornelissen, M. Zabeau, and P. Vos. 1998. Dissection of the fusarium $\mathrm{I}-2$ gene cluster in tomato reveals six homologs and one active gene copy. Plant Cell 10:1055-1068, https://doi. org/10.1105/tpc.10.6.1055.

Sol Genomics Network. 2019. International tomato genome sequencing project. 15 Oct. 2021 . < https://solgenomics.net>.

Stall, R.E. and J.W. Walter. 1965. Selection and inheritance of resistance in tomato to isolates of races 1 and 2 of the fusarium wilt organism. Phytopathology 55:1213-1215.

Volin, R.B. and J.P. Jones. 1982. A new race of fusarium wilt of tomato in Florida and sources of resistance. Proc. Florida State Hort. Soc. 95:268-270.

Tomato Genetics Resource Center. 2011. Seed stocks. 15 Oct. 2021. $<$ http://tgrc.ucdavis.edu $>$.

Walter, J.M., D.G.A. Kelbert, and N.C. Hayslip. 1961. Manapal a disease resistant tomato with desirable traits of Rutgers. Univ. Florida Agric. Exp. Sta. Circ. S-131. 


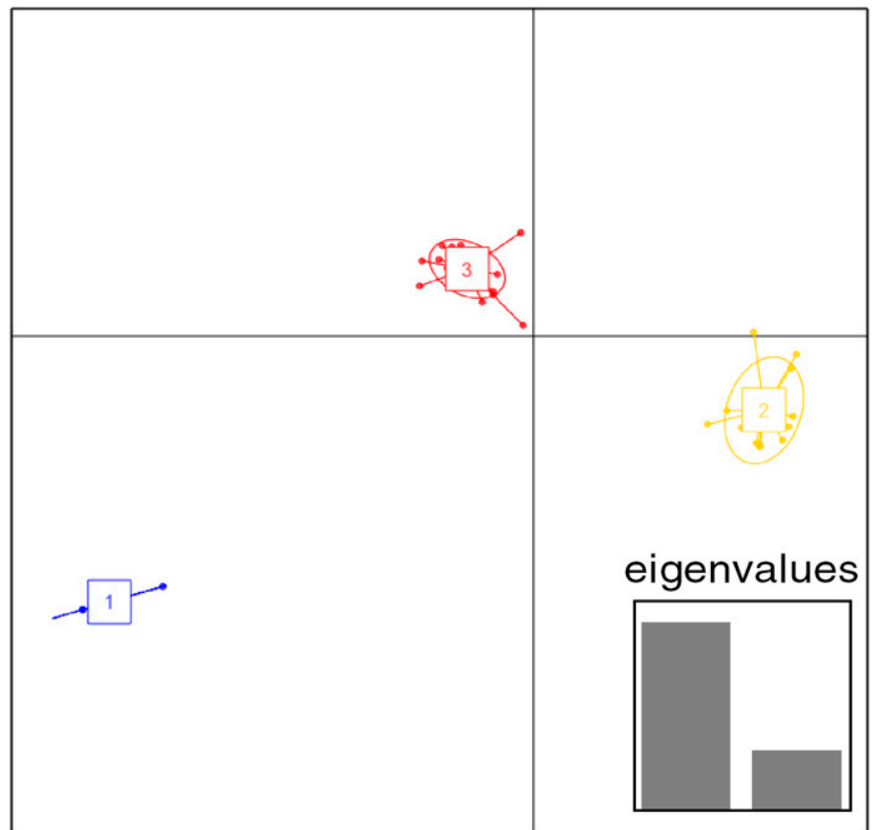

Supplemental Fig. 1. Genetic clusters inferred using discriminant analysis of principal components (DAPC). Clusters are shown by different colors and inertia ellipses, and dots represent individual Solanum pennellii accessions. The red, blue, and yellow groups represent the circle, empty triangle, and cross symbols in Fig. 1A, respectively. The inset indicates the eigenvalues of the analysis, which retains two principal components. 
Supplemental Table 1. Molecular markers used to detect the Solanum pennellii introgressions containing either I-3 or I-7.

\begin{tabular}{|c|c|c|c|c|c|}
\hline Gene & Marker $\left(5^{\prime}-3^{\prime}\right)$ & Type $^{z}$ & Restriction enzyme & Fragment size $(b p)^{y}$ & Physical position $^{\mathrm{x}}(\mathrm{Mb})$ \\
\hline \multirow[t]{3}{*}{$I-3$} & $\begin{array}{l}7 g 728 \\
\text { F: CACAACTGCCAAACATGACC } \\
\text { R: TAACCAAATTTATTGGCTTTTGGA }\end{array}$ & SCAR & none & 132 vs. 117 & 63.41 \\
\hline & $\begin{array}{l}7 g 923 \\
\text { F: ATTGGTTAAGTGAGACGTGTG } \\
\text { R: TAATGAATGCTGTGGCTAGGA }\end{array}$ & SCAR & none & 125 vs. 112 & 63.60 \\
\hline & $\begin{array}{l}S L G-1 \\
\text { F: CGTTCATAATCTGCTAAGTTTCTA } \\
\text { R: GGTTTCTATTACTCCAGCATCCTC }\end{array}$ & CAPS & RsaI & 296,170 vs. 466 & 63.63 \\
\hline \multirow[t]{2}{*}{$I-7$} & $\begin{array}{l}I-7 A \\
\text { F: TTTCTTATTGGAACCATC } \\
\text { R: GACTAAGTTTTGTGAGGCTA }\end{array}$ & PCR-HRM & none & 49 & 61.64 \\
\hline & $\begin{array}{l}I-7 B \\
\text { F: GATGCGTCAAGCTACTGAGTTCAC } \\
\text { R: TGCAGCTTTCTTATTGGAACCATC }\end{array}$ & PCR-HRM & none & 155 & 61.64 \\
\hline
\end{tabular}

${ }^{\mathrm{z}}$ Marker type included SCAR $=$ sequence characterized amplified region; CAPS = cleaved amplified polymorphic sequence; and PCR$\mathrm{HRM}=$ polymerase chain reaction, high-resolution melting marker.

${ }^{\text {y }}$ Size of detected amplicons/fragments: Solanum lycopersicum vs. S. pennellii.

${ }^{\mathrm{x}}$ Physical locations of the markers on chromosomes 7 and 8 for $I-3$ and $I-7$, respectively, are based on the tomato genome assembly, version SL2.50 (http://solgenomics.net/); "Mb" represents mega base. 
Supplemental Table 2. Sequence polymorphisms identified in 42 Solanum pennellii accessions using the genotyping by sequencing method.

\begin{tabular}{|c|c|c|c|}
\hline Accession & Mono-allelic SNPs ${ }^{2}$ & Heterozygous SNPs $^{y}$ & Heterozygosity $(\%)$ \\
\hline LA1943 & 29082 & 3005 & 10 \\
\hline LA716 & 33735 & 3756 & 11 \\
\hline LA2963 & 32445 & 4030 & 12 \\
\hline LA1946 & 32312 & 4878 & 15 \\
\hline LA2680 & 26057 & 3998 & 15 \\
\hline LA1724 & 26915 & 4147 & 15 \\
\hline LA1912 & 28018 & 4470 & 16 \\
\hline LA1733 & 26401 & 4292 & 16 \\
\hline LA750 & 29788 & 5019 & 17 \\
\hline LA1732 & 26039 & 4454 & 17 \\
\hline LA1911 & 27608 & 4759 & 17 \\
\hline LA1920 & 29121 & 5085 & 17 \\
\hline LA 1272 & 26602 & 4867 & 18 \\
\hline LA1942 & 28496 & 5227 & 18 \\
\hline LA1656 & 27952 & 5168 & 18 \\
\hline LA1340 & 27773 & 5182 & 19 \\
\hline LA1941 & 29920 & 5588 & 19 \\
\hline LA1522 & 28244 & 5366 & 19 \\
\hline LA1734 & 27143 & 5239 & 19 \\
\hline LA2560 & 26807 & 5218 & 19 \\
\hline LA1926 & 29588 & 5852 & 20 \\
\hline LA1297 & 27359 & 5497 & 20 \\
\hline LA1649 & 28608 & 5838 & 20 \\
\hline LA1275 & 25622 & 5282 & 21 \\
\hline LA1809 & 25876 & 5427 & 21 \\
\hline LA1282 & 27266 & 5936 & 22 \\
\hline LA1302 & 26471 & 5821 & 22 \\
\hline LA1735 & 25301 & 5667 & 22 \\
\hline LA1299 & 24157 & 5487 & 23 \\
\hline LA1693 & 28057 & 6451 & 23 \\
\hline LA2657 & 29175 & 6750 & 23 \\
\hline LA1657 & 25434 & 5912 & 23 \\
\hline LA1515 & 25313 & 5885 & 23 \\
\hline LA1367 & 25897 & 6022 & 23 \\
\hline LA1303 & 27058 & 6420 & 24 \\
\hline LA1273 & 24927 & 6053 & 24 \\
\hline LA1277 & 25012 & 6201 & 25 \\
\hline LA1356 & 25085 & 6327 & 25 \\
\hline LA1674 & 26713 & 6798 & 25 \\
\hline LA751 & 26671 & 6994 & 26 \\
\hline LA1376 & 24340 & 6526 & 27 \\
\hline LA1940 & 26384 & 7205 & 27 \\
\hline
\end{tabular}

${ }^{\mathrm{z}} \mathrm{SNP}=$ single nucleotide polymorphism.

${ }^{\mathrm{y}} \mathrm{SNP}$-containing positions that have a heterozygous sequence genotype. 